\title{
YAO'S MILLIONAIRES' PROBLEM AND DECOY-BASED PUBLIC KEY ENCRYPTION BY CLASSICAL PHYSICS
}

\author{
DIMA GRIGORIEV AND VLADIMIR SHPILRAIN
}

\begin{abstract}
We use various laws of classical physics to offer several solutions of Yao's millionaires' problem without using any one-way functions. We also describe several informationally secure public key encryption protocols, i.e., protocols secure against passive computationally unbounded adversary. This introduces a new paradigm of decoy-based cryptography, as opposed to "traditional" complexity-based cryptography. In particular, our protocols do not employ any one-way functions.
\end{abstract}

\section{INTRODUCTION}

The "two millionaires problem" introduced by Yao in [3] is: Alice has a private number $a$ and Bob has a private number $b$, and the goal of the two parties is to solve the inequality $a \leq b$ ? without revealing the actual values of $a$ or $b$, or more stringently, without revealing any information about $a$ or $b$ other than $a \leq b$ or $a>b$.

We note that all known solutions of this problem (including Yao's original solution) use one-way functions one way or another. (Informally, a function is one-way if it is efficient to compute but computationally infeasible to invert on "most" inputs.) The problem with those solutions is that it is still not known whether one-way functions actually exist, i.e., the functions used in the aforementioned solutions are just assumed to be one-way.

In this paper, we offer several very simple solutions of Yao's millionaires' problem without using any one-way functions, but using various laws of classical, everyday physics instead. We group our solutions in several sections of this paper, emphasizing not only different laws of physics employed, but also different underlying ideas. More specifically, in Section 3 we offer a solution that does not even employ any particular "laws" of physics, but just uses a simple mechanism, an elevator in a building. In Section 5, we employ the law of communicating vessels.

In Section 6, we offer public key encryption protocols that do not use any one-way functions and are secure against passive computationally unbounded adversary. Using one-way functions is considered mandatory in the "traditional", complexity-based, public key cryptography. (We suggest [5] as a general reference.) Security of our protocols, on the other hand, is decoybased (we explain below what it means) rather than complexity-based, and

Research of the second author was partially supported by the NSF grant CNS-1117675. 
this allows us to get rid of one-way functions together with problems that accompany this concept, including the lack of proof of the very existence of one-way functions.

We note that in our earlier paper [2], we have offered an encryption protocol with similar properties, based on principles of electrical engineering. That paper has invited unprofessional criticism of two kinds: (1) claims that our scheme is similar to schemes of Kish et al. (see [4]) because we "use electrical wires", like Kish does. (There are actually other similarities as well: for example, in our paper we use letters of the English alphabet, like Kish does); (2) suggesting incompetent "man-in-the-middle" attacks based on misunderstanding of principles of electrical engineering. Of course, the real purpose of both kind of criticism was to distract attention from the main point made in our paper [2], which is showing that there are secure encryption protocols that do not employ any one-way functions, but instead rely in their security on numerous "decoys" of the actual encrypted message, and this "decoy-based" cryptography presents an important alternative to the "traditional", complexity-based, cryptography. The work of Kish et al. does not use the idea of decoy; their protocols still rely in their security on (allegedly) one-way functions; it is just that the justification of their functions being one-way comes from physics (e.g. they use the second law of thermodynamics) rather than from mathematics.

An obvious advantage of decoy-based schemes is that they are secure even against computationally unbounded (passive) adversary, which cannot possibly be the case with complexity-based schemes. A disadvantage is that so far, our schemes (that employ principles of classical physics) require a physical medium, and this somewhat limits the range of transmission. It is therefore a central question whether or not some of our schemes based on laws of physics can be mimicked in the "traditional" scenario where communicating parties can only exchange sequences of bits with each other. Alternatively, one can look for a physical medium (waves, or photons, or something else) that would allow a long-range information transmission based on similar ideas. As we point out in our Section 6.4, we believe that radio waves can be used to provide such a long-range secure communication.

In this paper, we avoid using "electrical wires" and try to only use very simple physics in order to discourage ludicrous "attacks" and unprofessional criticism as above. Specifically, in Section 6.1, we just use classical Newton's laws of motion for public key encryption. This scenario, although not really practical, provides a crystal clear illustration of our "decoy" method. This particular protocol can only be used for communication over rather short distances, but on the other hand, it relays the idea of decoy-based encryption very clearly. It also clearly relays the particular way to implement the general idea of decoy that we use in the present paper, namely, combining private keys of Alice and Bob during the transmission. We note that in 
our paper [2], the implementation of the "decoy" idea was different: the adversary there faced an underdetermined system of equations, with multiple solutions for an unknown secret number.

In Section 6.2, building on the idea of combining private keys of the two parties, we describe a much more practical protocol (using acoustic waves) that allows communication over longer distances, although it still requires a physical medium, which limits the range. Finally, in Sections 6.3 and 6.4 we speculate on how to increase the range of transmission by using other kinds of waves.

\section{RANGe, PRIVATE SPACE, AND PRIVATE KEYS}

In the following three sections we address Yao's millionaires' problem. We assume here that $a$ and $b$ are positive integers, such that both are in the interval $\left[N_{1}, N_{2}\right]$ for some $N_{1}, N_{2} \in \mathbf{Z}_{+}$. Let $n=N_{2}-N_{1}$. We note that this $n$ can be made, by re-scaling, as large or as small as is convenient for a particular approach. For example, if $n$ is too large to handle by real-life tools, we can express $a$ as $a=a_{1} \cdot m+r_{a}$, and $b=b_{1} \cdot m+r_{b}$ for some public positive integer $m<n$ and positive integers $r_{a}, r_{b}<m$, and then compare $a_{1}$ to $b_{1}$, etc. One can think of this as representing $a$ and $b$ in, say, decimal form and then comparing them one digit at a time, going left to right.

An important part of our model is the concept of a private space. In the "traditional" setting, where the parties communicate over the Internet, the private space is a private computer that can, in particular, secretly generate private keys. Without this facility, there obviously would be no security. In our situation, where we use real-life tools, a private space for, say, Alice is typically a private room or other kind of container where nobody can observe her actions. Usually, the other party (Bob) also has a private space where nobody can observe his actions.

On the other hand, we assume that everybody (Alice, Bob, the eavesdroppers, if any) can observe (and measure) everything that is going on in the "public space", i.e., outside the union of Alice's and Bob's private spaces.

\section{3. "Elevator" SOlution}

This is logistically the simplest solution. Suppose there is an elevator building with at least $n=N_{2}-N_{1}$ floors. Alice positions herself on the floor number $a$, and Bob gets to the floor number $b$. After that, Bob takes an elevator (Bob's private space) going down, stopping at every floor. Alice is just watching the elevator doors on her floor, making sure that Bob does not see her when the elevator doors open (here is Alice's private space). If she ever sees the elevator doors open, she knows that Bob's number is larger. If not, then his number is smaller. Alternatively, when Bob gets to the ground floor, he can get in touch with Alice to find out whether she has seen the elevator doors open on her floor. That way, both parties will end up knowing whose number is larger. 


\section{4. "RACE TRACK" SOLUTION}

Here Alice and Bob run toward each other from the opposite ends of a race track of length $n=N_{2}-N_{1}$. Alice maintains the speed of $a \mathrm{~m} / \mathrm{s}$, and Bob maintains the speed of $b \mathrm{~m} / \mathrm{s}$. Whoever gets to the midpoint of the track first, leaves a mark there and runs back, knowing that he/she was faster, without knowing the actual speed of the other party. Then, when the other party gets to the midpoint, he/she will know that he/she was slower, again without knowing the actual speed of the other party. To arrange for their private space in this scenario, the parties have to put an inpenetrable fence across the track at the midpoint.

The "race track" idea can be actually implemented on a "usual" computer if we allow two different programs to work with the same file. That shared file would be a bit string of length $n$, with all bits initially equal to 1 . Alice provides a program that goes over this bit string left to right, replacing the current "1" symbol by "0" at the speed of one symbol per a time units. Bob provides a similar program going over the same bit string right to left, at the speed of one symbol per $b$ time units. When either program replaces $\frac{n}{2}$ symbols, it replaces the current symbol by "X" and stops. Whose program stops first has the smaller number. Note that both programs will have to use the computer's internal clock, which is a little unusual but not impossible.

\section{5. "Communicating Vessels" solution}

Here we have two communicating vessels. One of them, call it $U$, is in Alice's private space, and the other one, call it $V$, is in Bob's private space. These vessels are connected by a horizontal pipe attached to their bottoms.

In the beginning the system is "almost", but not completely, filled with water. Then Alice starts pumping the water out of her vessel at the speed of $a$ gallons (or whatever units) per second, while Bob starts pumping the water in his vessel at the speed of $b$ gallons per second. The parties are just watching whether the level of water is decreasing or increasing. If it is decreasing, then $a>b$; if it is increasing, then $a<b$.

\section{ENCRYPTION WITHOUT ONE-WAY FUNCTIONS}

In this section, we describe several encryption protocols, based on the same idea but on different laws of physics, whose security is decoy-based rather than complexity-based. These protocols are therefore secure even against computationally unbounded adversary.

6.1. Using laws of motion. We start with a very simple protocol that is not practical, but on the other hand, it relays the essence of the "decoy" idea very clearly. Here Alice and Bob are positioned at points A and B (respectively) of a long horizontal rod of known mass. Alice wants to transmit to Bob her secret number $F_{a}>0$. 
First we describe the idea informally. Alice applies, at the point A, a private force $F_{a}$ to the rod, moving it in the direction of Bob's point B. At the same time, Bob applies, at the point $\mathrm{B}$, his private force $F_{b}$ in the same direction. The total force acting on the rod therefore is $F=F_{a}+$ $F_{b}$. This total force is public information, i.e., anybody can measure it (by measuring the acceleration of the rod, for example) at will. However, only Bob knows $F_{b}$, so he can recover $F_{a}$ as $F-F_{b}$, while the adversary cannot. As far as the adversary is concerned, there are too many "decoy" values of $F_{a}$ because there are many ways to split public $F$ as a sum $F=$ $F_{a}+F_{b}$. However, for the "decoy" to work, Alice and Bob would have to synchronize the moment when they start applying their private forces because if somebody goes first, the adversary will be able to measure his/her force alone. Instead of trying to synchronize Alice and Bob, we offer here a more logistically feasible solution to this problem. Namely, Alice and Bob are going to gradually (and randomly) increase their forces until they stabilize at the values $F_{a}$ and $F_{b}$, respectively. This strategy is also useful in foiling some of the "man-in-the-middle" attacks, see discussion below, after the protocol description.

Here is a more formal description of our encryption protocol.

(1) Alice starts applying to the rod a force $F_{1}(t)$, which is a random function of time $t$, moving the rod in the direction of Bob's point B. Bob starts applying, in the same direction, a force $F_{2}(t)$, which is, too, a random function of time $t$. (We do not specify here what "a random function of $t$ " means; although this issue deserves special attention, addressing it here would lead us too far away from the mainstream of the paper.) When Bob starts applying his force, he tells Alice, publicly, that he is "in business"; this is needed to foil a "man-in-the-middle" attack by impersonating Bob (see discussion below).

(2) Eventually, after getting a confirmation that Bob is "in business", Alice stabilizes her force at $F_{a}$, and Bob stabilizes his force at $F_{b}$. Bob detects the stabilization by observing that the rod acceleration is not changing due to Alice's efforts for some fixed period of time, agreed upon by both parties up front.

(3) After the rod acceleration has stabilized, the force acting on it is $F=F_{a}+F_{b}$, so Bob recovers Alice's secret $F_{a}$ as $F_{a}=F-F_{b}$.

We note once again that security of this protocol is based on the presence of numerous "decoy" possibilities for $F_{a}$, resulting from the fact that there are many ways to split public $F$ as a sum $F=F_{a}+F_{b}$. Different combinations of possible values of these private keys can result in the same observable quantities in the public space. Thus, it is impossible for the adversary to single out, with non-negligible probability, the actual value of $F_{a}$ among all possible ones based on observations and measurements in the 
public space. We emphasize that what makes this possible is that the receiver (Bob) is able to influence the transmission of information from the sender (Alice) by using his private key, as opposed to the typical scenario in complexity-based cryptography where Bob, after having published his public $k e y$, is just "sitting there" waiting for information from Alice to arrive.

If the adversary is active (i.e., if she is not just observing and measuring but can interfere with the protocol itself), then she can, of course, just mess up the transmission by applying her own force to the rod, for example. This kind of interference cannot be avoided in any scenario including the "traditional" communication over the Internet where the Internet cable can be cut. However, this kind of interference is not so dangerous because the adversary does not get a hold of the secret. A more dangerous kind of interference, known as "man-in-the-middle" attack, is where the adversary is trying to impersonate the receiver, or sender, or both. In our scenario, the adversary can try to impersonate Bob (the receiver), but to compute the correct value of Alice's force $F_{a}$, the adversary then would have to somehow get rid of Bob's contribution. To prevent from being excluded from the protocol execution, Bob can just instruct Alice not to stabilize her force at $F_{a}$ until he tells her that he, too, has started to apply his force.

Thus, our protocol is also secure against some of the "man-in-the-middle" attacks, but we still encourage the reader to focus on the new and important paradigm of decoy-based cryptography, which provides security against passive computationally unbounded adversary. Of course, this protocol can only be used for communication over rather short distances, but on the other hand, it relays the idea of "decoy-based" encryption very clearly. In the following subsections, we use the same idea (combining private keys of Alice and Bob during the transmission) that we used in this simple protocol, but a different physical principle (superposition of waves), to allow communication over longer distances.

6.2. Using acoustic waves. Now, building on the same ideas, we are going to describe a much more practical protocol that allows communication over longer distances, although it still requires a physical medium, which limits the range.

Here Alice and Bob are going to generate acoustic waves in a common medium; one can think of an "old-fashioned", non-digital phone line, or some other acoustic waveguide. Alice and Bob are positioned at points A and $\mathrm{B}$ (respectively) of this common medium. Alice wants to transmit to Bob her secret number $A_{1}>0$, which is going to be the amplitude of her wave. The arrangement is similar to that in our previous subsection: Alice and Bob combine their waves (that have the same frequency and phase) to get a wave whose amplitude $A$ is the sum $A_{1}+A_{2}$ of the private amplitudes. Bob then recovers Alice's secret as $A_{1}=A-A_{2}$.

Here is a more formal description of this encryption protocol. 
(1) Alice and Bob publicly agree on the common frequency $\omega$ and phase $\varphi$ of their waves.

(2) Alice starts generating, at her point A, a wave with frequency $\omega$ and phase $\varphi$, while at the same time modulating the amplitude $A(t)$ as a random function of time $t$. Bob, too, starts generating his wave at his point $\mathrm{B}$, with frequency $\omega$ and phase $\varphi$, randomly modulating its amplitude. When Bob starts generating his wave, he tells Alice, publicly, that he is "in business".

(3) Eventually, after getting a confirmation that Bob is "in business", Alice stabilizes the amplitude of her wave at $A_{1}$, and Bob stabilizes the amplitude of his wave at $A_{2}$.

(4) After the amplitudes have stabilized, the amplitude of the superposition of Alice's and Bob's waves is $A_{1}+A_{2}$, so Bob recovers Alice's secret $A_{1}$ as $A_{1}=A-A_{2}$.

Security analysis here is the same as that in the previous subsection. Again, the main point is that there are numerous "decoy" possibilities for $A_{1}$, resulting from the fact that there are many ways to split the public amplitude $A$ as a sum $A=A_{1}+A_{2}$. Thus, different combinations of possible values of the private keys $A_{1}, A_{2}$ can result in the same observable quantities in the public space, so that even a computationally unbounded adversary cannot determine the actual secret $A_{1}$.

At this point we have to mention that the legend has it that the idea of using a superposition of waves to preserve privacy of communication (over the phone) was studied (secretly) in Bell Labs during World War II [6], as well as in the Soviet Union in the 1950s, and possibly also in the U.K. [1]. However, the idea was (allegedly) rejected because of insurmountable technological difficulties: in the pre-digital era, the "whole wave" (a person's voice), and not just its amplitude or frequency, would have to be retrieved in real time to make this idea useful in practice.

6.3. Using fiber optic. Communication using a fiber optic cable is one of the most widely used ways of transmitting information over the Internet. In this case, pulses of light are sent through an optical fiber. The light forms an electromagnetic carrier wave that is modulated to carry information. Thus, the same idea of superposition of waves (generated by the sender and by the receiver) that we described in Section 6.2 can be used in this situation as well.

6.4. Using radio waves. One can also use other kinds of waves, provided that Alice and Bob are connected by an appropriate waveguide (e.g. electromagnetic or optical). The challenge now is to get rid of a waveguide in order to increase the communication range dramatically, allowing secure communication between, say, the planet surface and a satellite. One of the most obvious ways to address this challenge would be using radio waves. It would be interesting to assess technological feasibility, in this context, of a 
protocol similar to that in our Section 6.2, but in any case, the theoretical idea of using superposition of two waves to hide the secret amplitude (or frequency) behind numerous decoys seems to be valid in this situation as well.

Acknowledgement. Both authors are grateful to Max Planck Institut für Mathematik, Bonn for its hospitality during the work on this paper. We are also grateful to Igor Monastyrsky for comments on physical aspects of our schemes.

\section{REFERENCES}

[1] J. Ellis, The possibility of secure non-secret digital encryption, CESG Report, January 1970.

[2] D. Grigoriev and V. Shpilrain, Secure information transmission based on physical principles, in: UCNC 2013, Lecture Notes Comp. Sc. 7956 (2013), 113-124.

[3] A. C. Yao, Protocols for secure computations (Extended Abstract), 23rd annual symposium on foundations of computer science (Chicago, Ill., 1982), 160-164, IEEE, New York, 1982.

[4] L. Kish et al., http : //www.ece.tamu.edu/ ${ }^{\sim}$ noise/research_files/research_secure.htm

[5] A. Menezes, P. van Oorschot, and S. Vanstone, Handbook of Applied Cryptography, CRC-Press 1996.

[6] Final Report on Project C43, Bell Telephone Laboratory, October 1944, p. 23.

CNRS, Mathématiques, Université de Lille, 59655, Villeneuve D’AscQ, FRANCE

E-mail address: dmitry.grigoryev@math.univ-lille1.fr

Department of Mathematics, The City College of New York, New York, NY 10031

E-mail address: shpil@groups.sci.ccny.cuny.edu 